\title{
Clinical utility of the neutrophil elastase inhibitor sivelestat for the treatment of acute respiratory distress syndrome
}

This article was published in the following Dove Press journal:

Therapeutics and Clinical Risk Management

5 August 2014

Number of times this article has been viewed

\author{
Naoki Aikawa' \\ Yasushi Kawasaki \\ 'School of Medicine, Keio \\ University, Tokyo, ${ }^{2}$ Ono \\ Pharmaceutical Co, Ltd, Osaka, \\ Japan
}

Correspondence: Yasushi Kawasaki Ono Pharmaceutical Co, Ltd,

I-8-2, Kyutaromachi, Chuo-ku,

Osaka 54I-8564, Japan

Tel +81662633902

Fax +8I 662632970

Email y.kawasaki@ono.co.jp

Abstract: Acute respiratory distress syndrome is a serious condition that can arise following direct or indirect lung injury. It is heterogeneous and has a high mortality rate. Supportive care is the mainstay of treatment and there is no definitive pharmacological treatment as yet. Sivelestat is a neutrophil elastase inhibitor approved in Japan and the Republic of Korea for acute lung injury, including acute respiratory distress syndrome in patients with systemic inflammatory response syndrome. The aim of this review is to examine the clinical utility of sivelestat in different disease states, using data from nonclinical and clinical studies. In nonclinical studies, sivelestat appears to show benefit in acute lung injury without inhibiting the host immune defense in cases of infection. Clinical studies do not yet provide a clear consensus. Phase III and IV Japanese studies have shown improvements in pulmonary function, length of intensive care unit stay, and mechanical ventilation, but a non-Japanese multicenter study did not demonstrate sivelestat to have an effect on ventilator-free days or 28-day all-cause mortality. Evidence of improvement in various parameters, including duration of stay in intensive care, mechanical ventilation, the ratio of partial pressure of arterial oxygen and fraction of inspired oxygen $\left(\mathrm{PaO}_{2} / \mathrm{F}_{1} \mathrm{O}_{2}\right.$ ratio $)$ ratio, and lung injury scores, has been shown in patients with sepsis or gastric aspiration, and following the surgical treatment of esophageal cancer. To date, there are no particular concerns regarding adverse events, and the available data do not suggest that sivelestat might worsen infections. One study has analyzed costeffectiveness, finding that sivelestat may reduce costs compared with standard care. The currently available evidence suggests that sivelestat may show some benefit in the treatment of acute lung injury/acute respiratory distress syndrome, although large, randomized controlled trials are needed in specific pathophysiological conditions to explore these potential benefits.

Keywords: acute lung injury, systemic inflammatory response syndrome, pharmacotherapy, ventilator free days, mortality

\section{Introduction}

Acute respiratory distress syndrome (ARDS) is a serious acute hypoxic condition that develops in patients with various underlying diseases and injuries. There is a relative lack of treatment options for ARDS, although new pharmacotherapeutic approaches are being trialled. The purpose of this review is to examine the clinical utility of one such approach to treating ARDS, namely sivelestat, a neutrophil elastase inhibitor, using data from nonclinical and clinical studies. The review also includes an examination of sivelestat's use in specific clinical conditions, such as sepsis, gastric aspiration, and following the surgical treatment of esophageal cancer.

\section{Clinical characteristics and epidemiology of ARDS}

ARDS exhibits a broad spectrum of clinical characteristics and distinct stages. In general, it is characterized by its acute onset, bilateral lung infiltrates on chest radiography, 
and decreased $\mathrm{PaO}_{2} / \mathrm{F}_{\mathrm{I}} \mathrm{O}_{2}(\mathrm{P} / \mathrm{F})$ ratio, which is not attributable to heart failure or volume overload. ${ }^{1}$

Accurate estimates of the mortality rate and incidence of ARDS are difficult because of the different definitions that exist and the heterogeneity of the disease. ${ }^{1}$ A review by Rubenfeld and Herridge in 2007 found reported incidences of ARDS ranging from 13.5 to 58.7 per 100,000 person-years, and reported mortality rates ranging from $34 \%$ to $57.9 \%$. $^{2}$

Both direct and indirect lung injury can result in the development of ARDS. Common direct causes include pneumonia and aspiration of stomach contents, and common indirect causes include sepsis, shock, and severe trauma. Overall, sepsis is associated with the highest risk of development of ARDS. ${ }^{1}$ Pathological findings specific to ARDS are referred to as diffuse alveolar damage, which includes intra-alveolar edema, fibrin deposition, hyaline membrane formation, and destruction of type I alveolar epithelial cells. ${ }^{3}$ Uncontrolled neutrophil-dominant inflammation and increased permeability of lung microvascular endothelium and alveolar epithelial cell layers are common pathophysiological features of ARDS, and clinically lead to nonhydrostatic pulmonary edema. ${ }^{4-8}$

\section{Diagnosis and treatment of ARDS}

The diagnosis of ARDS has long been based on the American-European Consensus Conference (AECC) definition, published in 1994, in which ARDS is defined as a subset of acute lung injury (ALI). ${ }^{9}$ In the AECC definition, ARDS is defined as having an acute onset, a $\mathrm{P} / \mathrm{F}$ ratio $\leq 200 \mathrm{mmHg}$, bilateral chest infiltrates on chest radiograph, and no evidence of left atrial hypertension. The AECC definition also incorporates ALI, defined using similar criteria but with less severe hypoxemia $(\mathrm{P} / \mathrm{F}$ ratio $\leq 300 \mathrm{mmHg}) .{ }^{9}$ This led to some confusion around the distinction between the terms ALI and ARDS, in addition to issues around the thresholds for defining acute onset, chest radiograph criteria, and distinguishing hydrostatic edema. ${ }^{10}$ In light of the issues around the clinical application of these criteria, a new definition, known as the Berlin definition, was proposed in 2012 to improve the specificity of the clinical diagnosis, although it is currently under discussion. ${ }^{10}$ In the Berlin definition, three categories of ARDS are defined based on the level of hypoxemia: mild ( $\mathrm{P} / \mathrm{F}$ ratio $>200$ to $\leq 300 \mathrm{mmHg}$ ), moderate $(\mathrm{P} / \mathrm{F}$ ratio $>100$ to $\leq 200 \mathrm{mmHg})$, and severe $(\mathrm{P} / \mathrm{F}$ ratio $\leq 100 \mathrm{mmHg})$, but the term ALI is not used. ${ }^{10}$ Because the clinical studies of sivelestat described in this review were conducted in individuals with ALI, including
ARDS based on the AECC definition, both terms (ALI and ARDS) are used where appropriate in this paper.

There have been extensive efforts to develop strategies for the treatment of ARDS, but, as yet, only low tidal volume ventilation and placement of the patient in a prone position have been shown to be effective. ${ }^{11,12}$ Whilst still high, it has been suggested that rates of mortality owing to ARDS might be falling gradually, possibly as a result of improvements in supportive care. ${ }^{1}$ Identifying and treating the underlying cause of ARDS is important, as is preventing hospitalacquired infections in patients. ${ }^{1}$

A number of pharmacological approaches have been attempted, such as the use of nitric oxide inhalation, neuromuscular blocking agents, and corticosteroids. These pharmacological approaches may be beneficial, but as yet there is no definitive treatment for ARDS. ${ }^{13-17}$ Regarding the use of corticosteroids, this has been investigated in various studies. ${ }^{14-16}$ A study of low-dose corticosteroids in late ARDS did not support the routine use of corticosteroids for persistent ARDS despite improvements in cardiopulmonary physiology, and starting therapy more than 2 weeks after the onset of ARDS was found to be possibly associated with an increase in the risk of death. ${ }^{15}$ Low doses of corticosteroids were associated with better outcomes in septic shock-associated early ARDS in nonresponders to the short cosyntropin stimulation test, but not in responders and not in patients with septic shock without ARDS. ${ }^{16}$

In addition to these supportive care and pharmacotherapeutic approaches, sivelestat, an inhibitor of neutrophil elastase, which is intravenously administered at $0.2 \mathrm{mg} / \mathrm{kg} /$ hour continuously for a maximum of 14 days, is available in Japan and the Republic of Korea for ALI (according to the AECC definition) with systemic inflammatory response syndrome (SIRS). ${ }^{18}$

\section{Nonclinical studies of sivelestat}

Neutrophil elastase is a serine protease produced by neutrophils. Its main physiological function is the degradation of phagocytosed foreign organic molecules within the cells. Extracellular neutrophil elastase is a highly destructive enzyme, capable of degrading a variety of extracellular proteins, including elastin, collagen, lung surfactant, and immunogloblins. ${ }^{4,19}$ In addition to its proteolytic activity, neutrophil elastase is also known to induce the production of inflammatory cytokines ${ }^{20}$ and mucin from epithelial cells. ${ }^{21}$ However, under physiological conditions, extracellular neutrophil elastase activity in the body is tightly regulated by endogenous protease inhibitors, such as $\alpha_{1}$-protease 
inhibitor. ${ }^{4,22}$ A possible role of neutrophil elastase in the pathogenesis of ALI/ARDS is shown in Figure $1 .{ }^{4}$ At inflammatory sites, the $\alpha_{1}$-protease inhibitor is inactivated by neutrophil-derived reactive oxygen species, thereby allowing extracellular neutrophil elastase to attack tissues. ${ }^{4,23,24}$

In animal models of ALI, it has been shown that various stimuli, such as endotoxin or viable Streptococcus pneumoniae, induce alveolar neutrophil infiltration and lung injury, associated with increased neutrophil elastase activity in the bronchoalveolar lavage fluid (BALF). ${ }^{25-33}$

Sivelestat, a selective neutrophil elastase inhibitor, was shown to inhibit the progression of lung injury in these models (Table 1). ${ }^{25-33}$ On the other hand, neutrophil elastase is essential for neutrophils to play a host defensive role. ${ }^{34}$ This fact raises the question of whether inhibition of neutrophil elastase activity might disrupt the host defense system, resulting in aggravation of infection.

In a hamster lung injury model induced by $S$. pneumoniae, sivelestat inhibited digestion of surfactant protein $\mathrm{D}$, an endogenous protein that protects against bacterial infection, and reduced the number of bacteria in BALF and lung tissues, indicating that, while inhibiting neutrophil elastase activity, sivelestat preserves the host immune defense system. ${ }^{33}$ Additionally, sivelestat did not influence the in vitro bactericidal capacity of neutrophils. ${ }^{35,36}$ Overall, inhibition of extracellular neutrophil elastase activity by sivelestat is beneficial in lung injury, including that induced by bacterial infection.

\section{Clinical studies of sivelestat Clinical efficacy}

Sivelestat is currently approved in Japan and the Republic of Korea for treating ALI, including ARDS in patients with SIRS, and its use has been investigated in several clinical studies (Table 2).

In a Phase III trial of 230 mechanically ventilated ALI patients with SIRS conducted in Japan, designed to confirm the efficacy and safety of sivelestat, sivelestat improved pulmonary function, reduced the duration of mechanical ventilation, and shortened stay in the intensive care unit (ICU). ${ }^{37}$ However, there was no significant effect of sivelestat on the 30-day survival rate. In an international multicenter (not including Japan), double-blind, placebo-controlled Phase II

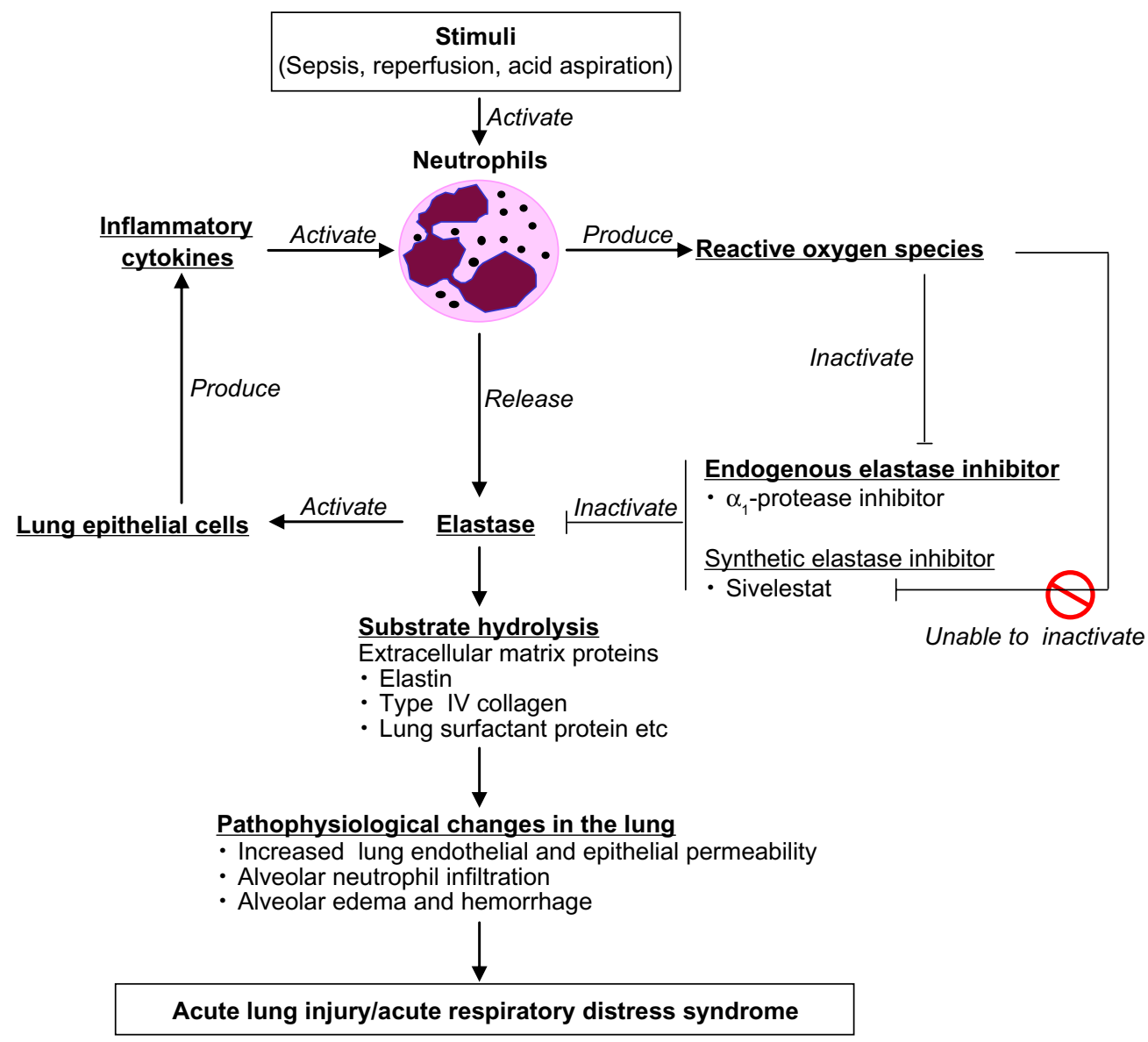

Figure I Possible role of neutrophil elastase in pathogenesis of acute lung injury/acute respiratory distress syndrome. 
Table I Nonclinical studies of sivelestat in lung injury models

\begin{tabular}{|c|c|c|c|c|}
\hline Reference & $\begin{array}{l}\text { Experimental disease } \\
\text { models }\end{array}$ & $\begin{array}{l}\text { Animal } \\
\text { species }\end{array}$ & Main outcome measures & Findings \\
\hline Kawabata et al ${ }^{26}$ & $\begin{array}{l}\text { Endotoxin inhalation- } \\
\text { induced lung injury }\end{array}$ & Hamster & $\begin{array}{l}\text { Protein concentration, leukocyte } \\
\text { count, and NE activity in BALF }\end{array}$ & $\begin{array}{l}\text { Sivelestat inhibited NE activity, and } \\
\text { decreased protein concentration and } \\
\text { leukocyte count in BALF. }\end{array}$ \\
\hline Hagio et $\mathrm{al}^{27}$ & $\begin{array}{l}\text { Complement-mediated } \\
\text { lung injury }\end{array}$ & Hamster & $\begin{array}{l}\text { Lung vascular permeability and } \\
\text { NE activity in plasma }\end{array}$ & $\begin{array}{l}\text { Sivelestat inhibited plasma NE activity and } \\
\text { prevented the increase in lung vascular } \\
\text { permeability. }\end{array}$ \\
\hline Sakamaki et $\mathrm{al}^{28}$ & $\begin{array}{l}\text { Endotoxin-induced lung } \\
\text { injury }\end{array}$ & Guinea pig & $\begin{array}{l}\text { Lung tissue wet to dry } \\
\text { weight ratio and lung vascular } \\
\text { permeability } \\
\text { Neutrophil count and NE } \\
\text { activity in BALF }\end{array}$ & $\begin{array}{l}\text { Sivelestat inhibited NE activity in BALF, } \\
\text { and prevented the increase in neutrophil } \\
\text { count in BALF, the lung tissue wet } \\
\text { to dry weight ratio and lung vascular } \\
\text { permeability. }\end{array}$ \\
\hline Kubo et $\mathrm{a}^{29}$ & $\begin{array}{l}\text { Endotoxin-induced lung } \\
\text { injury }\end{array}$ & Sheep & $\begin{array}{l}\text { Pulmonary artery pressure, } \\
\text { pulmonary vascular resistance, } \\
\text { and lung lymph flow } \\
\text { Neutrophil count in lung }\end{array}$ & $\begin{array}{l}\text { Sivelestat prevented the increase in } \\
\text { pulmonary artery pressure, pulmonary } \\
\text { vascular resistance, lung lymph flow, and } \\
\text { neutrophil count in lung. }\end{array}$ \\
\hline Kishima et $\mathrm{al}^{30}$ & $\begin{array}{l}\text { Ischemia and reperfusion } \\
\text { lung injury }\end{array}$ & Rabbit & $\begin{array}{l}\text { Filtration coefficient, shunt } \\
\text { fraction, and histology }\end{array}$ & $\begin{array}{l}\text { Sivelestat improved filtration coefficient, } \\
\text { shunt fraction, and histology. }\end{array}$ \\
\hline Miyazaki et al ${ }^{31}$ & TNF $\alpha$-induced lung injury & Rabbit & $\begin{array}{l}\text { Pulmonary artery pressure and } \\
\text { lung vascular permeability }\end{array}$ & $\begin{array}{l}\text { Sivelestat attenuated the increase in } \\
\text { pulmonary artery pressure and lung } \\
\text { vascular permeability. }\end{array}$ \\
\hline Hagio et $\mathrm{a}^{32}$ & $\begin{array}{l}\text { Acid aspiration-induced } \\
\text { lung injury }\end{array}$ & Hamster & $\begin{array}{l}\text { Survival rate } \\
\text { Protein concentration, leukocyte } \\
\text { count, and NE activity in BALF } \\
\mathrm{PaO}_{2}\end{array}$ & $\begin{array}{l}\text { Sivelestat reduced death by respiratory } \\
\text { failure, and improved the increase in BALF } \\
\text { parameters and the decrease in } \mathrm{PaO}_{2} \text {. }\end{array}$ \\
\hline Hagio et $\mathrm{a}^{33}$ & $\begin{array}{l}\text { Streptococcus pneumoniae- } \\
\text { induced pneumonia }\end{array}$ & Hamster & $\begin{array}{l}\text { Lung vascular permeability } \\
\text { Bacterial count, leukocyte count, } \\
\text { and NE activity in BALF }\end{array}$ & $\begin{array}{l}\text { Sivelestat inhibited NE activity and } \\
\text { reduced the increase in lung vascular } \\
\text { permeability without affecting leukocyte } \\
\text { count. Sivelestat inhibited digestion } \\
\text { of surfactant protein D and facilitated } \\
\text { bacterial clearance. }\end{array}$ \\
\hline
\end{tabular}

Abbreviations: BALF, bronchoalveolar lavage fluid; NE, neutrophil elastase; TNF, tumor necrosis factor; $\mathrm{PaO}_{2}$, partial pressure of arterial oxygen.

study (STRIVE [Sivelestat Trial in ALI Patients Requiring Mechanical Ventilation]) that included 492 mechanically ventilated patients with ALI, there was no effect of sivelestat on the primary endpoints of ventilator-free days or 28-day all-cause mortality. ${ }^{38}$

In a postmarketing open-label, nonrandomized, multicenter clinical study of 404 Japanese ALI patients with SIRS and 177 controls, designed to re-evaluate the efficacy and safety of sivelestat in real-life clinical settings in Japan, the sivelestat group showed a significant improvement in the primary endpoint of number of ventilator-free days compared with the control group. ${ }^{39}$ This postmarketing study used AECC diagnostic criteria $^{9}$ but a post hoc analysis of the data using the Berlin definition ${ }^{10}$ produced similar results (data not published).

These studies show contradictory results. Potential reasons for the discrepancy might be that the Japanese clinical studies had younger subjects with less severe respiratory function (Figure 2) and less organ derangement, and excluded burns or trauma patients. In addition, the Japanese clinical studies defined SIRS as an inclusion criterion, while the
STRIVE study did not. ${ }^{37,38}$ Therefore, the difference in study populations may have influenced the study results. Following the findings of the STRIVE study, the package insert in Japan was revised to include the precautions that sivelestat is not to be used in patients with four or more organ failures or in patients with ALI resulting from burns or trauma. ${ }^{18}$

A systematic review and meta-analysis of eight randomized controlled trials of sivelestat for the treatment of ALI/ ARDS has been conducted. ${ }^{40}$ The meta-analysis included one multinational trial and seven Japanese trials. The primary outcome measure was mortality within 28-30 days of randomization. The authors did not find any evidence that sivelestat improved the primary outcome measure of 28-30day mortality (relative risk $0.95,95 \%$ confidence interval 0.72-1.26). Although sivelestat improved oxygenation on day 3 , it did not alter the duration of mechanical ventilation. As stated by the authors, possible limitations of the metaanalysis included its small sample size and lack of complete blinding in most of the included studies. In addition, the meta-analysis excluded trials examining the prophylactic 


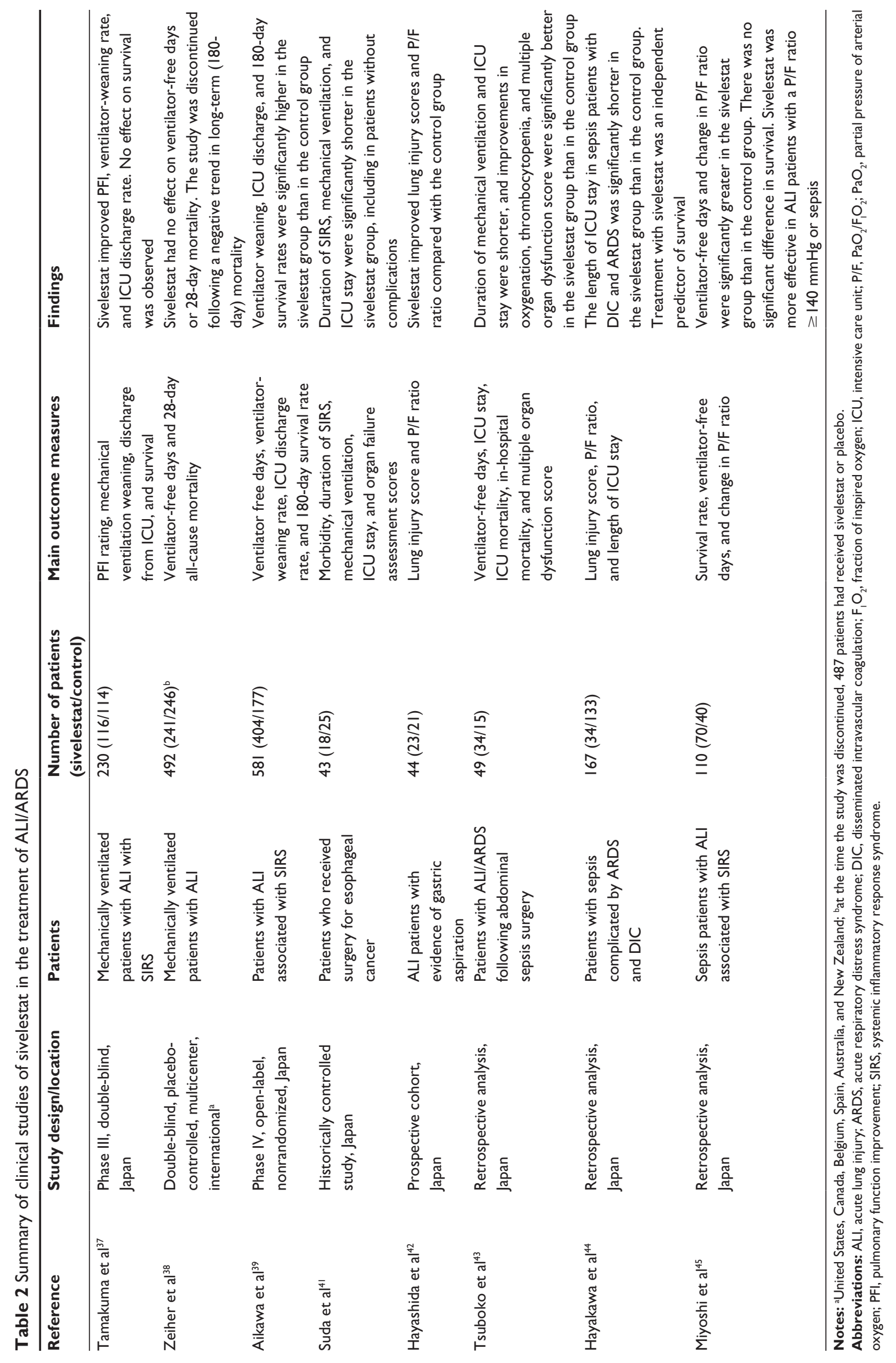




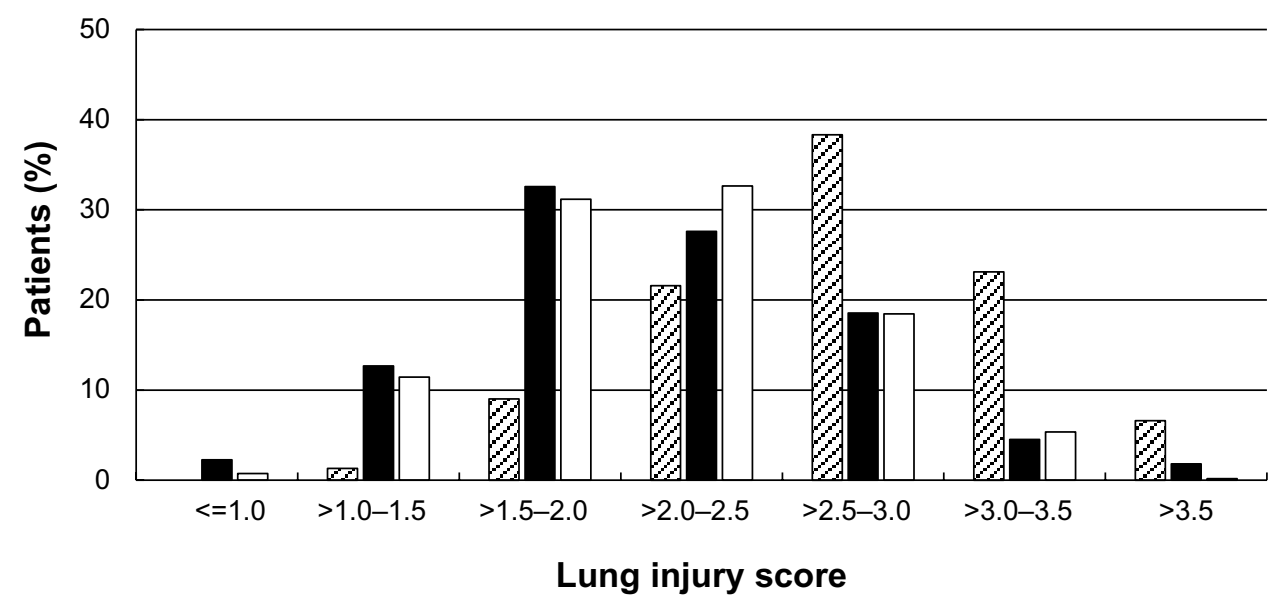

Figure 2 Lung injury scores of patients included three different clinical studies for sivelestat.

Notes: The white bars represent a postmarketing study in Japan ${ }^{39}(\mathrm{n}=542)$, the black bars represent a Phase III study in Japan ${ }^{37}(\mathrm{n}=22 \mathrm{I})$, and the hatched bars represent the STRIVE (Sivelestat Trial in ALI Patients Requiring Mechanical Ventilation) study ${ }^{38}(n=454)$. Data from a post hoc analysis of patient data by Ono Pharmaceutical Co Ltd (Osaka, Japan; data on file, 2008).

use of sivelestat. ${ }^{40}$ The results highlight the need to examine the effect of sivelestat on mortality in larger, double-blind, randomized controlled trials in the future.

A possible explanation for the failure of other approaches may be that previous clinical trials included all patients who met the AECC diagnostic criteria, without the careful exclusion of patients with other diseases. Although these studies do not provide a general consensus on the clinical use of sivelestat, to date it is one of the few drug therapies for the treatment of ALI and ARDS.

In general, evaluating the effectiveness of pharmacotherapy for ARDS is difficult because of the nature of this multifactorial disease. Clinical findings and time courses vary among ALI patients, including those with ARDS, depending on the time after onset and the underlying diseases and injuries. In addition, the pathophysiological conditions and responses to treatment may be heterogeneous, and proving the pragmatic endpoint of landmark all-cause mortality rates in ARDS patients is difficult.

\section{Efficacy in specific clinical conditions}

Although the indication for sivelestat in Japan and the Republic of Korea is ALI (according to the AECC definition) in patients with SIRS, the clinical effectiveness of sivelestat in ALI/ARDS caused by various conditions has been evaluated in several studies. ${ }^{41-45}$

Postoperative complications following surgical treatment of esophageal cancer are common. Therefore, the use of sivelestat in ARDS following esophageal cancer surgery was examined in a Japanese study. ${ }^{41}$ Morbidity, duration of SIRS, mechanical ventilation, ICU stay, and organ failure assessment scores were compared between the sivelestat group $(n=18)$ and a historical control group $(n=25)$ that underwent transthoracic esophagectomy. Duration of SIRS, mechanical ventilation, and ICU stay were significantly shorter in the sivelestat group, including in patients without complications. ${ }^{41}$

Gastric aspiration is a common direct cause of ALI and ARDS. A prospective study of 44 patients with aspiration pneumonia (sivelestat group, $\mathrm{n}=23$; control group, $\mathrm{n}=21$ ) was conducted at two university hospitals in Japan. Lung injury score and $\mathrm{P} / \mathrm{F}$ ratio on day 7 after admission to ICU were the primary outcome measures, and sivelestat showed favorable effects on lung injury score and the $\mathrm{P} / \mathrm{F}$ ratio compared with contols. $^{42}$

Three studies have examined the use of sivelestat in ALI/ARDS associated with sepsis. ${ }^{43-45}$ The first looked at a cohort of 49 Japanese patients (sivelestat group, $n=34$; control group, $\mathrm{n}=15$ ) with ALI/ARDS who had been surgically treated for abdominal sepsis. The group receiving sivelestat had a significantly reduced number of days of mechanical ventilation and ICU stay. In addition, significant improvements in oxygenation, thrombocytopenia, and multiple organ dysfunction score were noted. ${ }^{43}$

The second study was a retrospective data analysis of 167 patients (sivelestat group, $n=34$; control group, $\mathrm{n}=133$ ) with sepsis complicated by ARDS and disseminated intravascular coagulation. The ICU stay was shorter in the sivelestat group, and sivelestat was found to be an independent predictor of survival in this group of patients with sepsis complicated by ARDS and disseminated intravascular coagulation. ${ }^{44}$ 
Finally, a retrospective study examined the use of sivelestat in 110 sepsis patients (sivelestat group, $n=70$; control group, $\mathrm{n}=40$ ) with ALI associated with SIRS. The number of ventilator-free days and the increase in $\mathrm{P} / \mathrm{F}$ ratio were significantly greater in the sivelestat group than in the control group, but there was no difference in overall survival between the two groups. ${ }^{45}$ The authors concluded that, on the basis of these findings, randomized controlled trials designed to determine whether sivelestat is beneficial for ALI patients with sepsis are warranted.

In the light of the heterogeneity of ALI/ARDS, confirming the efficacy of sivelestat in patients with specific pathophysiological states is of benefit. However, since all the above studies were nonrandomized, there are limitations in interpretation of the results. Further studies, including randomized controlled trials, are necessary to evaluate the efficacy of sivelestat in patients with specific clinical conditions.

\section{Safety of sivelestat}

A summary of adverse events based on clinical studies and postmarket surveillance is shown in Table 3. To date, available clinical study data, including for the STRIVE study and the related postmarketing study, indicate no particular concerns regarding adverse events. ${ }^{38,39}$

In the STRIVE study, while there was no difference between sivelestat and placebo in terms of all-cause 28-day mortality, a negative trend in long-term (180-day) mortality prompted the Data and Safety Monitoring Board to recommend suspension of enrollment and discontinuation of the study. Blinded review of a sample of the cases of death from each group could not identify the reason for this increase in late mortality. ${ }^{38}$ Long-term survival was not a primary endpoint of the study, so further studies are needed to investigate this. There were no significant differences between the sivelestat and placebo groups in terms of adverse events, including serious infectious adverse events. ${ }^{38}$

In the Japanese postmarketing study, ${ }^{39}$ which was conducted at the request of the Pharmaceuticals and Medical Devices Agency to re-evaluate the safety and efficacy of sivelestat, there was no significant difference between groups with regard to the incidence of serious adverse events related to infection, and no serious adverse infectious event was considered to be related to the study drug. The incidence of adverse events was significantly lower in the sivelestat group than in the control group, and the 180-day survival rate was significantly higher in the sivelestat group than in the control group. ${ }^{39}$ The cause of the difference in 180-day survival is not clear, but differences in study populations between the STRIVE study and the Japanese postmarketing study might have influenced the results.

These clinical data do not indicate that sivelestat can worsen infection, and this finding is further supported by a study in an animal model of $S$. pneumoniae-induced lung injury, in which bacterial counts in BALF and lung interstitial tissue were reduced by sivelestat, while the host immune response was preserved. . $^{33,38,39}$

Table 3 Summary of adverse events of sivelestat ${ }^{46}$

\begin{tabular}{|c|c|c|}
\hline \multirow[t]{2}{*}{ Disorders } & \multicolumn{2}{|l|}{ Incidence } \\
\hline & $1 \%-10 \%$ & $<\mathbf{1} \%$ \\
\hline Hypersensitivity & - & Rash \\
\hline \multirow[t]{5}{*}{ Hepatobiliary disorders } & Blood bilirubin increased & Urobilinogen urine increased \\
\hline & Alanine aminotransferase increased & Blood lactate dehydrogenase \\
\hline & Aspartate aminotransferase increased & increased \\
\hline & Gamma-glutamyltransferase increased & \\
\hline & Blood alkaline phosphatase increased & \\
\hline \multirow[t]{5}{*}{ Blood and lymphatic system disorders } & - & Eosinophilia \\
\hline & & Thrombocytopenia \\
\hline & & Thrombocytosis \\
\hline & & Anemia \\
\hline & & Hemorrhage \\
\hline \multirow[t]{4}{*}{ Renal and urinary disorders } & - & Blood urea nitrogen increased, \\
\hline & & Blood creatinine increased \\
\hline & & Polyuria \\
\hline & & Protein urine \\
\hline \multirow[t]{3}{*}{ Others } & - & Hyperkalemia \\
\hline & & Protein total decreased \\
\hline & & Injection site phlebitis \\
\hline
\end{tabular}




\section{Cost-effectiveness of sivelestat}

A cost-minimization analysis of sivelestat in treating patients with ALI associated with SIRS caused by infection has been conducted in Japan using data from a Phase III Japanese trial. ${ }^{47,48}$ Although the periods on mechanical ventilation and in ICU were shorter for the sivelestat group than for the control group by only 2 days and 1.8 days, respectively, these differences were significant in terms of reduction in medical costs. Based on 2001 values, from the Japanese health care payer perspective, the analysis suggested that sivelestat may reduce costs compared with standard care in this group of patients. ${ }^{47}$

\section{Summary}

In summary, ARDS is a serious condition with a high mortality rate. It is heterogeneous and manifests as various morbid states depending on the underlying causes. In animal models, sivelestat appears to show benefit in the treatment of acute lung injury, without inhibiting host immune defense in cases of infection. ${ }^{25-33}$ Clinical studies do not yet provide general consensus on the clinical use of sivelestat. Two Japanese studies, one Phase III and one Phase IV, have together shown improvements in pulmonary function, and reductions in the length of ICU stay and duration of mechanical ventilation on sivelestat. ${ }^{37,39}$ However, in STRIVE, a multicenter study conducted outside Japan, sivelestat did not affect the number of ventilator-free days or 28-day all-cause mortality. ${ }^{38}$ This difference in outcomes may be the result of a difference in study populations. In addition, the fact that STRIVE was terminated because of adverse 180-day survival rates may have influenced the findings. Other studies have investigated the use of sivelestat in ARDS caused by specific conditions, such as sepsis and gastric aspiration, and following the surgical treatment of esophageal cancer. ${ }^{41-45}$ Such studies are useful in clarifying the clinical use of sivelestat, and further studies in groups of patients with specific causes of lung injury will be beneficial. In terms of short-term safety, there are no particular concerns regarding adverse events, and the available data do not suggest that sivelestat might worsen infections. ${ }^{33,38,39}$ One study in ICU patients with ALI associated with SIRS analyzed cost-effectiveness, and suggested that sivelestat may reduce costs when compared with standard care. ${ }^{47}$

Based on the evidence available to date, the neutrophil elastase inhibitor sivelestat appears to show some benefit for the treatment of ALI/ARDS. However, these findings are based on limited data in humans, and it must be noted that larger studies are needed to further explore the effect on mortality. Although there is some evidence for the efficacy of sivelestat in specific clinical conditions, further studies, particularly randomized controlled trials, are needed to add to the current knowledge regarding the efficacy and safety of this agent in the management of ALI/ARDS.

\section{Disclosure}

NA has received fees for manuscript writing and lectures from Ono Pharmaceutical Co, Ltd. YK is an employee of Ono Pharmaceutical Co, Ltd.

\section{References}

1. Ware LB, Matthay MA. The acute respiratory distress syndrome. $N$ Engl J Med. 2000;342(18):1334-1349.

2. Rubenfeld GD, Herridge MS. Epidemiology and outcomes of acute lung injury. Chest. 2007;131(2):554-562.

3. Tomashefski JF Jr. Pulmonary pathology of the acute respiratory syndrome. Clin Chest Med. 2000;21(3):435-466.

4. Kawabata K, Hagio T, Matsuoka S. The role of neutrophil elastase in acute lung injury. Eur J Pharmacol. 2002;451(1):1-10.

5. Idell S, Kucich U, Fein A, et al. Neutrophil elastase-releasing factors in bronchoalveolar lavage from patients with adult respiratory distress syndrome. Am Rev Respir Dis. 1985;132(5):1098-1105.

6. Rocker GM, Wiseman MS, Pearson D, et al. Diagnostic criteria for adult respiratory distress syndrome: time for reappraisal. Lancet. 1989;1(8630):120-123.

7. Donnelly SC, MacGregor I, Zamani A, et al. Plasma elastase levels and the development of the adult respiratory distress syndrome. Am J Respir Crit Care Med. 1995;151(5):1428-1433.

8. Tönz M, Mihaljevic T, von Segesser LK, et al. Acute lung injury during cardiopulmonary bypass. Are the neutrophils responsible? Chest. 1995;108(6):1551-1556.

9. Bernard GR, Artigas A, Brigham KL, et al. The American-European Consensus Conference on ARDS. Definitions, mechanisms, relevant outcomes, and clinical trial coordination. Am J Respir Crit Care Med. 1994;149(3 Pt 1):818-824.

10. ARDS Definition Task Force; Ranieri VM, Rubenfeld GD, Thompson BT, et al. Acute respiratory distress syndrome: the Berlin Definition. JAMA. 2012;307(23):2526-2533.

11. The Acute Respiratory Distress Syndrome Network. Ventilation with lower tidal volumes as compared with traditional tidal volumes for acute lung injury and the acute respiratory distress syndrome. $N \mathrm{Engl}$ J Med. 2000;342(18):1301-1308.

12. Guérin C, Reignier J, Richard JC, et al. Prone positioning in severe acute respiratory distress syndrome. $N$ Engl J Med. 2013;368(23): 2159-2168.

13. Spieth PM, Zhang H. Pharmacological therapies for acute respiratory distress syndrome. Curr Opin Crit Care. 2014;20(1):113-121.

14. Wheeler AP, Bernard GR. Acute lung injury and the acute respiratory distress syndrome: a clinical review. Lancet. 2007;369(9572): $1553-1564$

15. Wajanaponsan N, Reade MC, Milbrandt EB. Steroids in late ARDS? Crit Care. 2007;11(4):310.

16. Aldabbagh T, Milbrandt EB, Linden PK. Steroids in early ARDS? Crit Care. 2007;11(3):308.

17. Papazian L, Forel JM, Gacouin A, et al. Neuromuscular blockers in early acute respiratory distress syndrome. $N$ Engl J Med. 2010;363:1107-1116.

18. Japanese Respiratory Society for ARDS. [Clinical practice guideline for acute lung injury and acute respiratory distress syndrome]. Nihon Kokyuki Gakkai Zasshi. 2010;Suppl:1-101. Japanese. 
19. Havemann K, Gramse M. Physiology and pathophysiology of neutral proteinases of human granulocytes. Adv Exp Med Biol. 1984;167:1-20.

20. Bedard M, McClure CD, Schiller NL, et al. Release of interleukin-8, interleukin-6, and colony-stimulating factors by upper airway epithelial cells: implications for cystic fibrosis. Am J Respir Cell Mol Biol. 1993;9(4):455-462.

21. Kohri K, Ueki IF, Nadel JA. Neutrophil elastase induces mucin production by ligand-dependent epidermal growth factor receptor activation. Am J Physiol Lung Cell Mol Physiol. 2002;283(3): L531-L540.

22. Travis J, Salvesen GS. Human plasma proteinase inhibitors. Annu Rev Biochem. 1983;52:655-709.

23. Weiss SJ. Tissue destruction by neutrophils. $N$ Engl J Med. 1989;320(6):365-376.

24. Sandhaus RA, Turino G. Neutrophil elastase-mediated lung disease. COPD. 2013;10 Suppl 1:60-63.

25. Kawabata K, Hagio T, Matsuoka S. [Pharmacological profile of a specific neutrophil elastase inhibitor, sivelestat sodium hydrate]. Nihon Yakurigaku Zasshi. 2003;122(2):151-160. Japanese.

26. Kawabata K, Hagio T, Matsumoto S, et al. Delayed neutrophil elastase inhibition prevents subsequent progression of acute lung injury induced by endotoxin inhalation in hamsters. Am J Respir Crit Care Med. 2000;161(6):2013-2018.

27. Hagio T, Nakao S, Matsuoka H, et al. Inhibition of neutrophil elastase activity attenuates complement-mediated lung injury in the hamster. Eur J Pharmacol. 2001;426(1-2):131-138.

28. Sakamaki F, Ishizaka A, Urano T, et al. Effect of a specific neutrophil elastase inhibitor, ONO-5046, on endotoxin-induced acute lung injury. Am J Respir Crit Care Med. 1996;153(1):391-397.

29. Kubo K, Kobayashi T, Hayano T, et al. Effects of ONO-5046, a specific neutrophil elastase inhibitor, on endotoxin-induced lung injury in sheep. J Appl Physiol. 1994;77(3):1333-1340.

30. Kishima H, Takeda S, Miyoshi S, et al. Microvascular permeability of the non-heart-beating rabbit lung after warm ischemia and reperfusion: role of neutrophil elastase. Ann Thorac Surg. 1998;65(4):913-918.

31. Miyazaki Y, Inoue T, Kyi M, et al. Effects of a neutrophil elastase inhibitor (ONO-5046) on acute pulmonary injury induced by tumor necrosis factor alpha (TNF alpha) and activated neutrophils in isolated perfused rabbit lungs. Am J Respir Crit Care Med. 1998;157(1): 89-94.

32. Hagio T, Matsumoto S, Nakao S, et al. Elastase inhibition reduced death associated with acid aspiration-induced lung injury in hamsters. Eur $J$ Pharmacol. 2004;488(1-3):173-180.

33. Hagio T, Kishikawa K, Kawabata K, et al. Inhibition of neutrophil elastase reduces lung injury and bacterial count in hamsters. Pulm Pharmacol Ther. 2008;21(6):884-891.

34. Belaaouaj A, McCarthy R, Baumann M, et al. Mice lacking neutrophil elastase reveal impaired host defense against gram negative bacterial sepsis. Nat Med. 1998;4(5):615-618.
35. Akimoto A, Mori H, Namba T, et al. The effects of sivelestat sodium hydrate (ONO-5046 Na) on neutrophil phylaxis function in vitro. Jpn Pharmacol Ther. 2003;31:85-91.

36. Zeiher BG, Matsuoka S, Kawabata K, Repine JE. Neutrophil elastase and acute lung injury: prospects for sivelestat and other neutrophil elastase inhibitors as therapeutics. Crit Care Med. 2002;30(5):S281-S287.

37. Tamakuma S, Ogawa M, Aikawa N, et al. Relationship between neutrophil elastase and acute lung injury in humans. Pulm Pharmacol Ther. 2004;17(5):271-279.

38. Zeiher BG, Artigas A, Vincent JL, et al. Neutrophil elastase inhibition in acute lung injury: results of the STRIVE study. Crit Care Med. 2004;32(8):1695-1702.

39. Aikawa N, Ishizaka A, Hirasawa $\mathrm{H}$, et al. Reevaluation of the efficacy and safety of the neutrophil elastase inhibitor, sivelestat, for the treatment of acute lung injury associated with systemic inflammatory response syndrome; a phase IV study. Pulm Pharmacol Ther. 2011;24(5):549-554.

40. Iwata K, Doi A, Ohji G, et al. Effect of neutrophil elastase inhibitor (sivelestat sodium) in the treatment of acute lung injury (ALI) and acute respiratory distress syndrome (ARDS): a systematic review and meta-analysis. Intern Med. 2010;49(22):2423-2432.

41. Suda K, Kitagawa Y, Ozawa S, et al. Neutrophil elastase inhibitor improves postoperative clinical courses after thoracic esophagectomy. Dis Esophagus. 2007;20(6):478-486.

42. Hayashida K, Fujishima S, Sasao K, et al. Early administration of sivelestat, the neutrophil elastase inhibitor, in adults for acute lung injury following gastric aspiration. Shock. 2011;36(3):223-227.

43. Tsuboko Y, Takeda S, Mii S, et al. Clinical evaluation of sivelestat for acute lung injury/acute respiratory distress syndrome following surgery for abdominal sepsis. Drug Des Devel Ther. 2012;6:273-278.

44. Hayakawa M, Katabami K, Wada T, et al. Sivelestat (selective neutrophil elastase inhibitor) improves the mortality rate of sepsis associated with both acute respiratory distress syndrome and disseminated intravascular coagulation patients. Shock. 2010;33(1):14-18.

45. Miyoshi S, Hamada H, Ito R, et al. Usefulness of a selective neutrophil elastase inhibitor, sivelestat, in acute lung injury patients with sepsis. Drug Des Devel Ther. 2013;7:305-316.

46. Elaspol ${ }^{\circledR}$ (sivelestat sodium hydrate) [package insert]. Osaka, Japan: Ono Pharmaceutical Co., Ltd; 2002. Available from http://www.info.pmda. go.jp/downfiles/ph/PDF/180188_3999422D1020_1_09.pdf. Accessed July 10, 2014. Japanese.

47. Aikawa N, Fujishima S, Kobayashi M, et al. Cost-minimisation analysis of sivelestat for acute lung injury associated with systemic inflammatory response syndrome. Pharmacoeconomics. 2005;23(2):169-181.

48. Tamakuma S, Shiba T, Hirasawa H, et al. [A phase III clinical study of a neutrophil elastase inhibitor; ONO-5046 $\mathrm{Na}$ in SIRS patients]. Rinshoiyaku. 1998;14:289-318. Japanese.
Therapeutics and Clinical Risk Management

\section{Publish your work in this journal}

Therapeutics and Clinical Risk Management is an international, peerreviewed journal of clinical therapeutics and risk management, focusing on concise rapid reporting of clinical studies in all therapeutic areas, outcomes, safety, and programs for the effective, safe, and sustained use of medicines. This journal is indexed on PubMed Central, CAS,

\section{Dovepress}

EMBase, Scopus and the Elsevier Bibliographic databases. The manuscript management system is completely online and includes a very quick and fair peer-review system, which is all easy to use. Visit http://www.dovepress.com/testimonials.php to read real quotes from published authors. 